\title{
Analysis of Visual Function and the Effectiveness of Assistive Devices in a Glaucoma Cohort at a Tertiary Eye Hospital
}

\section{Priyanka Sudhakar $^{1}$ and Meena G Menon ${ }^{2 *}$}

${ }^{1}$ Glaucoma Fellow, Sankara Eye Hospital, Bangalore, Karnataka, India

${ }^{2}$ Head of Glaucoma Services, Sankara Eye Hospital, Bangalore, Karnataka, India

*Corresponding Author: Meena G Menon, Head of Glaucoma Services, Sankara Eye

Hospital, Bangalore, Karnataka, India.
Received: April 28, 2021

Published: May 26, 2021

(C) All rights are reserved by Priyanka

Sudhakar and Meena G Menon.

\section{Abstract}

Background: Since quality of life depends fairly on individual ability to perform everyday tasks, a better understanding of the different types of visual disability that patients with glaucoma encounter, becomes almost imperative to the clinician in tailoring their approach to delivering superior visual care.

Aims: To analyse effect of glaucoma on parameters of visual function, across 3 grades of disease severity and to evaluate efficacy of assistive devices in functionally rehabilitating these individuals.

Settings and Design: Observational 3 group cohort clinical assessment study of 120 eyes of 60 patients diagnosed with glaucoma, between August 2017 and January 2018.

Materials and Methods: The disease severity of these eyes were graded. Visual function was subjectively assessed with a questionnaire and objectively by colour vision, contrast sensitivity, visual field, binocular function. Data thus obtained was analysed against each grade of disease severity. Based on subjective assessment; assistive devices (magnifying spectacles, magnifiers, illumination devices, Fresnel prisms) were tried and their effectiveness analysed.

Statistical Analysis: Paired and unpaired t test and ANOVA.

Results: 61 patients (40 males and 21 females), with mean age of 60.43 years were enrolled. Majority (69.16\%) had Primary open angle glaucoma. Most of them had moderate to severe (29.6\%) and severe (27.7\%) glaucoma. While there was no significant difference among groups in terms of colour vision loss, significant loss of contrast sensitivity in $65.7 \%$ and stereoacuity in $66.6 \%$ was observed. Of the 19 eyes evaluated for low vision, 14 of them were given trial of magnifiers and illumination devices for reading difficulty, other 5 were given trial of Fresnel prism. All of them improved with the respective devices.

Conclusion: Glaucoma affects colour vision, contrast sensitivity and stereopsis, in addition to visual field and acuity, thus contributing to poor quality of vision. One cannot ignore the possibility of including evaluation of these parameters of visual function as part of the routine glaucoma work-up, for a holistic approach to visual care Fresnel prism as a potential visual aid, subject to further study. Keywords: Visual Function; Fresnel Prism; Assistive Devices; QoL

\section{Introduction}

Glaucoma is a progressive optic neuropathy that can culminate in irreversible vision loss, crippling the quality of life (QoL) of af- fected patients in various ways [1]. The fact that it is the most common cause of irreversible blindness globally only makes it alarming. However, the concern with respect to the course of this disease 
and its clinical implication does not limit only to the possibility of blindness as an end-stage sequel, but also to the poor quality of vision, that significantly deteriorates their visual experience and indirectly, their quality of life. This is a significant public health concern as those with low vision report more difficulty performing activities of daily living (ADL). Functional complaints of glaucoma patients with low vision are primarily related to reading, walking/ mobility, and driving. Of more concern in these patients is independent mobility as there is strong evidence to show that glaucoma patients are at increased risk of fall, motor vehicle collision, and subsequent morbidity $[2,3]$. In this subset of patients with visual impairment, in whom any further medical or surgical intervention is of any likely benefit, addressing their visual function deficits, by visually rehabilitating them is gaining importance. In-addition to the morbidity, glaucoma also adds to the economic burden of the individual as well as the society. It could impact the direct costs in the form of ocular medications, physician and hospital visits, glaucoma-related procedures, transportation and nursing home care, especially in late stages of the disease [4]. This only amplifies the greater need to understand the disease profile and address this problem more holistically.

Since QoL depends fairly on individual ability to perform everyday tasks, a better understanding of the different types of visual disability that patients with glaucoma encounter, becomes almost imperative to the clinician in tailoring their approach to delivering superior visual care.

Addressing this aspect, we have incorporated a questionnaire, in order to quantify QoL of patients with impaired visual function. It aids in furnishing additional information to the conventional measures of visual function, such as VA, CS and VF examination. In addition, it can also serve as an adjunctive tool to determine the effects of low-vision rehabilitation in glaucomatous patients.

Though studies in the past have revealed the visual function deficits associated with glaucomatous eyes, not only is there paucity of literature on the significance and correlation of these parameters with disease severity, but also, most of the available data in this regard, is based out of a western study population. In addition, despite the knowledge of poor quality of vision in patients with glaucoma, there is insufficient data on the potential role of assistive devices in the armamentarium of glaucoma management. In our study, we attempt to analyse these areas in a subset of Indian eyes and highlight other possible areas of clinical relevance, subject to further research.

\section{Materials and Methods}

Following clearance from the ethical committee, patients with glaucoma (known case or newly diagnosed), visiting the glaucoma clinic during June 2016 through June 2018, were included in this prospective, observational, cohort study. Patients included in the study were those aged 18 - 75 years and diagnosed with primary open angle glaucoma (POAG) or primary angle closure glaucoma (PACG) or pseudoexfoliative glaucoma (PXFG) or steroid induced glaucoma (SIG). While patients with neovascular glaucoma (NVG), malignant glaucoma, glaucoma secondary to inflammatory conditions, associated retinal or corneal disease, past history of refractive eye surgery, aphakia, and associated cataract were excluded.

An informed consent was obtained and a brief not of patient's demographics was made, relevant clinical history taken. All subjects underwent vision, refraction, anterior segment examination with slit-lamp, Goldmann applanation tonometry, gonioscopy and fundus examination with plus 90D lens.

The eyes that met both the exclusion and inclusion criteria were selected for further assessment.

Once we made the preliminary diagnosis, the severity of disease was graded based on the ICD-9 staging definitions:

1. Mild - Optic nerve abnormalities consistent with glaucoma but no visual field abnormalities on any white-on-white visual field test, or abnormalities present only on short-wavelength automated perimetry or frequency-doubling perimetry.

2. Moderate - Optic nerve abnormalities consistent with glaucoma and glaucomatous visual field abnormalities in one hemi field, and not within 5 degrees of fixation.

3. Severe - Optic nerve abnormalities consistent with glaucoma and glaucomatous visual field abnormalities in both hemi fields, and/or loss within 5 degrees of fixation in at least one hemi field.

We included the following tests as part of objective assessment of visual function:

- Visual acuity: While Log MAR chart was used to record the uncorrected and best-corrected visual acuity for distance, the near acuity was measured using $\mathrm{N}$-chart, which was subsequently converted to Log MAR for analysis.

- Colour vision: Assessed with Ishihara 21 plate test. 
- $\quad$ Contrast sensitivity: The Pelli-Robson chart was used to test each eye separately with the non-tested eye occluded. Values obtained were categorized into 2 groups- normal and abnormal based on a study done in 2001 by Maija Mäntyjärvi., et al. [5], which provided age-matched normal values as mentioned in table 1.

- Binocular function: Assessed based on the Worth-four-dottest and Stereopsis.

- Visual field: Recorded using the Humphrey's single field analysis using SITA standard strategy, with 30-2 / 24-2, and an additional 10-2 protocol, in necessary cases, to look for macular split. The field defects were graded according to the modified Hodapp-Parish-Anderson criteria (by Mills., et al. [6]) into six categories- minimal, early, moderate, severe, advanced and end-stage.

\begin{tabular}{|l|c|c|}
\hline \multicolumn{1}{|c|}{ Age range (years) } & OD & \multicolumn{2}{c|}{ OS } \\
\hline $20-39$ & \multicolumn{2}{|c|}{$1.65-1.95$} \\
\hline $40-59$ & $1.65-1.80$ & $1.5-1.80$ \\
\hline$>/=60$ &
\end{tabular}

Table 1: Normal contrast sensitivity (age matched) - Maija Ma"ntyjärvi., et al.

Following this, patients were made to fill-out a visual function questionnaire, as part of subjective evaluation of their visual function. This questionnaire was validated by running a statistical analysis with a reliability of $82.6 \%$.

We ran a trial of assistive devices to address the functional deficits as obtained on the subjective and objective evaluation of visual function. Magnifying spectacles, magnifiers (hand-held, stand, digital), illumination devices and Fresnel prism, were the assistive devices included in the study. To evaluate the effectiveness of magnifying spectacles, magnifiers and illumination devices, $\mathrm{N}$-chart was used and near visual acuity was recorded as the smallest print that could be read (recorded in N notation), at a specified distance, both before and after the trial. In case of an illumination device, we regulated the brightness using a lux meter. The smallest print the patient could read at a comfortable and optimum illumination was noted.

Fresnel prisms are thin prisms made of polyvinyl chloride with multiple individual prisms aligned in series. There are several techniques using Fresnel prisms. We have used the technique, which involves applying base out only to the temporal side (Figure 1). This brings objects centrally from the periphery approximately $1^{\circ}$ for every two prism dioptres of Fresnel prism. Arc perimetry was performed, by asking the patient to look at the central white dot, while a bright object was advanced slowly from the temporal side towards the central white dot, until the patient appreciated it. This point was noted to be the extent of his visual field. Similarly, this was repeated for the superior, inferior and nasal fields as well. This point noted is also indicative of where the central edge of the prism was to be applied. The prism was then cut and applied to the patient's spectacle lenses, base out. The patient is then encouraged to make eye movements into the prism to experience the change in field position and then return to the central area of the lens without the prism. Following this, the patient's mobility is assessed by asking him/her to stand and walk, scanning into the prism and then back to the central non-prism portion [7].

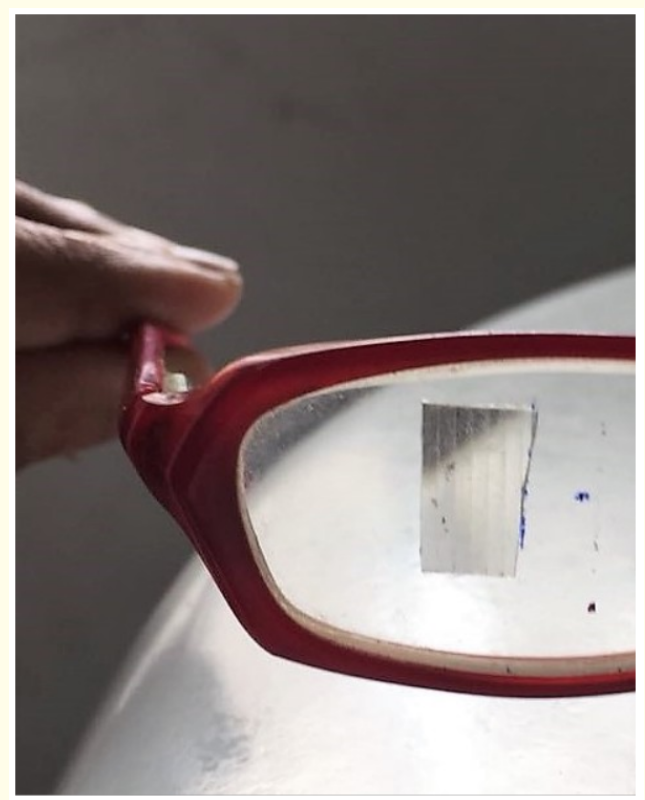

Figure 1: Fresnel prism.

\section{Results}

One hundred and twenty eyes of 61 patients ( 40 males and 21 females) were analysed. Majority, 83 of 120 eyes, had POAG. Most of the eyes in the patients in each of the different types of glaucoma had moderate $(34.2 \%)$ to severe $(47.5 \%)$ grades of glaucoma, as illustrated in table 2 . We categorized visual acuity based on ICD- 
10 classification of visual impairment. While a striking majority $80.3 \%$ (97 of 120 eyes) had mild or no impairment, only 10 of the 120 eyes had moderate impairment (low vision), which also correlated with the severity of the disease in these patients ( 9 of 10 had severe glaucoma). Table 3 shows the colour vision analysis. Majority (56.6\%) had normal colour vision. However, 47 of 120 eyes (39.2\%) had defective colour vision. The defective colour vision co-related with the severity of disease, with 41 of these 47 eyes (87.2\%) having severe glaucoma. 55\% had reduced contrast sensitivity, of whom $68.2 \%$ had severe glaucoma, as laid out in table 4. Stereopsis and W4DT, being tests of binocular function, could be assessed for 61 and 54 patients respectively. Though 43 of them (70.5\%) had loss of stereo-acuity, the co-relation of this defect with disease severity was not found to be statistically significant, possibly due to the small sample size. Majority of the patients maintained fusion for both distance and near ( 45 and 50 respectively). Table 5 illustrates analysis of stereopsis results. Since most of the eyes (57 of 120) had severe glaucoma, most frequently noted field defects were severe and advanced ones. Most of those with mild glaucoma had a minimal defect, while a majority of those with moderate grade had an early defect.

\begin{tabular}{|l|c|c|c|c|}
\hline \multirow{2}{*}{ Diagnosis } & \multicolumn{3}{|c|}{ Severity (N = 120) } & \multirow{2}{*}{ Mild } \\
\cline { 2 - 4 } & n (\%) & $\begin{array}{c}\text { Moderate } \\
\text { n (\%) }\end{array}$ & $\begin{array}{c}\text { Severe } \\
\text { n (\%) }\end{array}$ & P value \\
\hline POAG & $12(14.5 \%)$ & $31(37.3 \%)$ & $40(48.2 \%)$ & \\
PACG & $5(26.3 \%)$ & $1(5.3 \%)$ & $13(68.4 \%)$ & \\
NTG & $5(31.2 \%)$ & $9(56.2 \%)$ & $2(12.5 \%)$ & \\
PXF glaucoma & $0(0 \%)$ & $0(0 \%)$ & $2(100 \%)$ & \\
Total & $22(18.3 \%)$ & $41(34.2 \%)$ & $57(47.5 \%)$ & 0.006 \\
\hline
\end{tabular}

Table 2: Type of glaucoma and grades of severity.

\begin{tabular}{|l|c|c|c|c|c|}
\hline \multirow{2}{*}{$\begin{array}{c}\text { Colour } \\
\text { vision } \\
\text { grade }\end{array}$} & N & Mild & Moderate & Severe & \multirow{2}{*}{ P value } \\
\cline { 3 - 5 } & & $\mathbf{n}(\%)$ & $\mathbf{n}(\%)$ & (\%) & \\
\hline$>17$ & 68 & $21(30.9)$ & $32(47.1)$ & $\begin{array}{c}15 \\
(22.1)\end{array}$ & \\
14 to 16 & 5 & $0(0)$ & $4(80.0)$ & $1(20.0)$ & $<0.0001$ \\
$<=13$ & 47 & $1(21.0)$ & $5(10.6)$ & $\begin{array}{c}41 \\
(87.2)\end{array}$ & \\
\hline
\end{tabular}

Table 3: Analysis of colour vision.

\begin{tabular}{|l|c|c|c|c|c|}
\hline \multirow{2}{*}{$\begin{array}{c}\text { Contrast } \\
\text { Sensitivity } \\
\text { Grade }\end{array}$} & \multirow{2}{*}{$\mathbf{N}$} & \multicolumn{3}{|c|}{ Severity } & \\
\cline { 3 - 5 } & & Mild & Moderate & Severe & P value \\
Normal & 54 & $17(31.5)$ & $24(43.6)$ & $13(23.6)$ & $<$ \\
Abnormal & 66 & $4(6.1)$ & $17(25.8)$ & $45(68.2)$ & 0.0001 \\
\hline
\end{tabular}

Table 4: Analysis of contrast sensitivity results.

\begin{tabular}{|l|c|c|c|c|}
\hline \multirow{2}{*}{$\begin{array}{c}\text { Stereopsis } \\
\text { grade }\end{array}$} & \multicolumn{3}{|c|}{ Severity (N = 61) } & \multirow{2}{*}{ Pild } \\
\cline { 2 - 4 } & n (\%) & $\begin{array}{c}\text { Moderate } \\
\text { n (\%) }\end{array}$ & $\begin{array}{c}\text { Severe } \\
\text { n (\%) }\end{array}$ & \\
\hline$<40$ & $3(16.7)$ & $5(27.8)$ & $10(55.6)$ & \\
$>=40$ & $2(4.7)$ & $14(32.6)$ & $27(62.8)$ & \\
Total & $5(8.2)$ & $19(31.1)$ & $37(60.7)$ & 0.296 \\
\hline
\end{tabular}

Table 5: Comparative analysis of stereopsis.

The questionnaire, comprising 17 questions, evaluated areas of visual satisfaction, distance vision, near vision, visual field, sensory adaptation and colour vision. Of these, the questions which significantly correlated with the severity of glaucoma $(p<0.05)$, addressed:

- Visual satisfaction

- Distance and near vision

- Visual field

- Colour vision.

Of the 120 eyes assessed, 30 had low vision, defined according to the WHO 1992 working group classification. Of the 30 eyes noted to have low vision, only 10 patients (19 eyes) were willing for an evaluation of low vision. The improvement noted by an increase in visual field noted on arc perimetry, on a trial of Fresnel prism, was found to be statistically significant $(p=0.042)$. However, the improvement with magnifiers/ illumination devices, were not found to be statistically significant $(\mathrm{p}=0.153)$.

\section{Discussion}

We are all aware that glaucoma is a silent thief of vision. Various studies, done worldwide, have shown its possible effect on other parameters of visual function as well, thus impairing the over-all quality of vision in these patients [2,3,8,8-21]. We have, in our 
study, analysed both subjectively and objectively, this effect of glaucoma on visual function, and the possible role of assistive devices in them. The mean age of patients was $60.43 \pm 13.05$ years. Forty of the 61 patients were males, with a male: female ratio of 1.9:1. Most of the eyes had POAG (83 of 120 eyes), while 19 eyes had PACG, 16 had NTG and two had PXFG. In a study by Parrish., et al. [20] results were similar with 61\% having POAG as diagnosis and 38\% having glaucoma other than POAG. Fifty-seven of the 120 eyes (47.5\%) had severe glaucoma, while 41 eyes (34.2\%) had moderate and 22 eyes (18.3\%) had mild glaucoma. In our cohort, 80.8\% (97 of 120 eyes) had mild to no visual impairment with best glass correction. Most of these eyes belonged to the moderate and severe groups of glaucoma (40 and 37 eyes respectively) [ $p=0.007$ ]. In a study by CX Hu., et al. [22] in 2014, 75\% had a BCVA of 20/20 in their better eye and $91 \%$ had BCVA of $20 / 30$ or better. The mean visual acuity was $0.3 \pm 0.62$. In a study by Bassi., et al. [13] the mean obtained was $0.8 \pm 0.03$. Though the degree of visual acuity impairment was significantly comparable with the severity of visual field loss $(\mathrm{p}=$ 0.001 ), the correlation between BCVA as such and visual field loss was found to be less $(r=-0.528)$. When compared to the recent study by Jessica Liu., et al. [23] a very closely comparable result was noted ( $r=-0.51, p<0.001)$. Hawkins., et al. [14] in 2003, they found a similarly less correlation $(r=-0.322)$. In a comparison between BCVA and colour vision, though 97 of 120 eyes (80.8\%) had normal colour vision as well as mild or no visual impairment, it was interesting to note that most of the eyes with defective colour vision (30 of $47,63.8 \%$ ) also had a mild or no visual impairment [ $p=0.012$ ]. In a similar comparison between BCVA and contrast sensitivity in these eyes, a significant correlation was seen $(r=-0.286, p=0.027)$, with a further regression analysis showing that, with worsening of BCVA by one unit, the contrast reduced by 0.251 units [ $p=0.027$ ] This was highly comparable to a study by Jessica Liu., et al. [23], in 2018, who noted a similar significant correlation $(\mathrm{r}=-0.22, \mathrm{p}=$ $0.03)$.

In our study, we found that the mean colour vision was 13.06 \pm 8 .83. It was noted to be normal in majority of the patients ( 68 of $120,56.7 \%$ ). However, 47 of the 120 eyes, who did have a definite defective colour vision, (patients reading less than 13 plates in the Ishihara test), 41 of whom had severe glau coma [ $<<0.0001]$. It also correlated with the visual fields, which showed that majority (37 of 68 eyes) of those with normal colour vision had no or minimal to early field defects, while majority of those with defective colour vision (31 of 47 eyes) had an advanced field defect (according to the Hodapp-Parrish grading of field loss] $[\mathrm{p}<0.001]$. However, with the analysis of correlation between colour vision versus field loss $(\mathrm{r}=-.449, \mathrm{p}<0.001)$. This was in sync with the results in a study by MP Bambo., et al. [18] in 2016, who found that there was no significant correlation between the visual field indices and the colour vision abnormalities.

In our cohort, the mean contrast sensitivity was $1.3 \pm 0.47$. This was comparable to a recent study by Jessica Liu., et al. [23], where the mean was noted to be $1.38 \pm 0.17$ and to study by Richman., et al. [12] where the mean was $1.28 \pm 0.33$. Hawkins., et al. [14], in 2003 , also showed comparable result, with the mean contrast being $1.44 \pm 0.20$. We observed that, most of the eyes ( 66 of 120 eyes, $55 \%$ ) had a statistically significant abnormal contrast [ $<0.0001$ ]. In-addition, 45 of the 66 eyes (68.2\%) with decreased contrast, belonged to severe glaucoma group. In a comparison between contrast sensitivity and visual field loss, there was a better correlation between ( $\mathrm{r}=.225, \mathrm{p}=0.019$ ) as compared to the correlation between colour vision and field loss. This result was comparable to those obtained by Hawkins., et al. [14] and Wilensky., et al. [8] who reported a significant correlation between the mean deviation as measured with the Humphrey perimeter and the Pelli-Robson contrast sensitivity scores $(r=0.56, p<0.001, n=127 ; r=0.59$, $p<0.001, n=120$, respectively). We also studied the relation between contrast and visual acuity, as noted above had a significant correlation.

In our study, the mean stereopsis was $172.69 \pm 156.29$. Bassi., et al. [13] in 1991, in their study, noted the mean stereopsis to be $147.90 \pm 34.00$. Most of the patients had a defective stereopsis (43 of 55 patients, $78.1 \%$ ), which again, correlated with increasing severity of disease. This however was noted to be not statistically significant $(p=0.296)$. On comparing stereopsis with other parameters of visual function, such as field loss, there was a significant correlation $(r=0.294, p=0.03$ ). This was comparable to a study by Lakshmanan., et al. [16] in 2013, where a statistically better correlation was obtained $(\mathrm{p}<0.001)$.

Most of the eyes had early (30 eyes) or advanced (31 eyes) field loss, which correlated with increasing severity of disease (moderate and severe glaucoma, respectively) [p $<0.0001]$. As noted previously, the correlation of colour vision was found to be weaker as compared to that of contrast with the field loss, which was in accordance with similar studies by Wilensky., et al. [8] and Hawkins., et al [14].

In terms of visual satisfaction, most patients ( 43 of $61,70.5 \%, \mathrm{p}$ $=0.05$ ) were concerned about the possibility of worsening of glau- 
coma. Twenty-five of them (58.1\%) had severe glaucoma while 18 of them (41.8\%) had moderate glaucoma. Majority of the patients in our study had a difficulty in reading. In terms of distance vision ( $p=0.023$ ), 63.9\% of the patients had difficulty reading bus numbers, while in terms of near vision ( $p=0.001), 73.8 \%$ had difficulty reading newsprint. The subjective evaluation done by Aspinall., et al. [9], in 2008, also found reading and seeing details as the most common difficulties faced by people with glaucoma. In terms of patients' perception of visual field, $68.9 \%$ of the patients (42 of 61 , $\mathrm{p}<0.0001$ ) neither faced any difficulty in crossing roads nor did they have a problem of bumping into objects (51 of $61,83.6 \%, p$ $=0.021$ ). However, as a measure of stereopsis, majority of them (32 of $61,52.4 \%, p=0.004$ ) had difficulty in perceiving a dip in the ground or steps, which correlated with their disease severity, with 26 of them having severe glaucoma. However, we also found that a significant proportion (27 of $61,44.3 \%$ ) of the patients had no difficulty in terms of depth perception. 14 of them (51.8\%) belonged to the moderate glaucoma group. This data obtained from the questionnaire, is comparable to a study by CX Hu., et al. [22], done in 2014, which suggested that in contrast to the traditional view of glaucoma, loss of peripheral vision is not the most common symptom reported by the patients.

Of the 19 eyes evaluated for low vision, while 14 of them were given a trial of magnifiers and illumination devices to address their near reading difficulty, the other five were given a trial of Fresnel prism. We found that all of them improved with the devices used that were used to aid in near vision. This included, magnifying devices, illumination devices and magnifying spectacles. This result was similar to a recent study by Yogesh Patodia., et al. [24] who observed a statistically significant improvement in the reading ability in their cohort of 17 patients. Our result however, was not statistically significant. Of the five eyes evaluated for Fresnel prism, we observed that all of them showed an increase in their horizontal visual field, as measured on arc perimetry. Since the field of view improved significantly in these patients, the results thus obtained were statistically significant.

Though objective assessment of visual function showed impaired colour vision, contrast sensitivity and stereopsis in these patients, the respective assistive devices to aid in improvement of these conditions, like yellow chrome glasses, contrast enhancing measures and devices were not included in the list of assistive devices for trial. In addition, the evaluation of assistive devices in these patients was only a one-time assessment with no follow-up, thus other confounding factors like compliance, effect of these devices on other parameters of visual function come into play, and the cost-effectiveness of these devices. Furthermore, the smaller sample size could possibly be the reason for limitation in analysis of binocular function in these patients. So also, the trial of Fresnel prism, thus emphasizing the need for further studies with a bigger sample size to ascertain the results obtained.

\section{Conclusion}

Based on the results of our study, we conclude that, glaucoma affects not just the visual field, and visual acuity, as has been conventionally acknowledged, but also other important parameters of visual function like colour vision, contrast sensitivity and stereopsis, in a significant way. This accounts for the poor quality of vision in these patients. We could also consider the improvement in visual field with the aid of Fresnel prism as a potential area, for further study and evaluation, in providing an improved field of view to these patients. In conclusion, from our study we hereby, would like to highlight the multidimensional impact of glaucoma on visual function, which could possibly be areas of visual rehabilitation of these patients, subject to further study. Thus, one cannot ignore the possibility of including the evaluation for measures of these visual function parameters as part of the routine glaucoma work-up, in providing a more holistic approach to visual care to these patients.

\section{Clinical Significance}

Though there is an increasing awareness of glaucoma in the population at large, the concern is more about complete loss of sight in the long run, than the silent impact of the disease on quality of vision, due to apparently subtle changes in the components contributing to good quality of vision. This study highlights the different areas of visual function that are compromised as a consequence of glaucoma, thereby paving way for further research on ways to functionally rehabilitate these patients to a better quality of living.

\section{Bibliography}

1. Weinreb RN and Khaw PT. "Primary open-angle glaucoma". Lancet 363 (2004).

2. Gutierrez P., et al. "Influence of glaucomatous visual field loss on health-related quality of life". Archives of Ophthalmology 115 (1997).

3. Ramrattan RS., et al. "Prevalence and causes of visual field loss in the elderly and associations with impairment in daily func- 
tioning: the Rotterdam Study". Archives of Ophthalmology 119 (2001).

4. Varma R., et al. "An assessment of the health and economic burdens of glaucoma". American Journal of Ophthalmology 152 (2011).

5. Mäntyjärvi M and Laitinen T. "Normal values for the Pelli-Robson contrast sensitivity test". Journal of Cataract and Refractive Surgery 27 (2001): 261-266.

6. Mills RP and Drance SM. "Esterman disability rating in severe glaucoma”. Ophthalmology 93 (1986).

7. Schacknow PN and Samples JR. "The glaucoma book: a practical, evidence-based approach to patient care”. Springer (2010).

8. Wilensky JT and Hawkins A. "Comparison of contrast sensitivity, visual acuity, and Humphrey visual field testing in patients with glaucoma". Transactions of the American Ophthalmological Society American Ophthalmological Society 99 (2001): 213217.

9. Aspinall PA., et al. "Evaluation of Quality of Life and Priorities of Patients with Glaucoma". Investigative Opthalmology and Visual Science 49 (2008).

10. Burton R., et al. "Glaucoma and Reading". Optometry and Vision Science 89 (2012): 1282-1287.

11. Ross JE., et al. "Contrast sensitivity and visual disability in chronic simple glaucoma". The British Journal of Ophthalmology 68 (1984): 821-827.

12. Richman J., et al. "Importance of Visual Acuity and Contrast Sensitivity in Patients With Glaucoma". Archives of Ophthalmology 128 (2010): 1576-1582.

13. Bassi CJ and Galanis JC. "Binocular Visual Impairment in Glaucoma”. Ophthalmology 98 (1991): 1406-1411.

14. Hawkins AS., et al. "Comparison of contrast sensitivity, visual acuity, and Humphrey visual field testing in patients with glaucoma". Journal of Glaucoma 12 (2003).

15. Ramakrishnan R., et al. "Glaucoma in a rural population of southern India". Ophthalmology 110 (2003): 1484-1490.

16. Lakshmanan Y and George RJ. "Stereoacuity in mild, moderate and severe glaucoma". Ophthalmic and Physiological Optics 33 (2013).
17. Ramulu P. "Glaucoma and disability: which tasks are affected, and at what stage of disease?" Current Opinion in Ophthalmology 20 (2009): 92-98.

18. Bambo MP., et al. "Evaluation of Contrast Sensitivity, Chromatic Vision, and Reading Ability in Patients with Primary Open Angle Glaucoma”. Journal of Ophthalmology (2016): 1-6.

19. Lin S., et al. "Predicting Visual Disability in Glaucoma With Combinations of Vision Measures". Translational Vision Science and Technology. The Association for Research in Vision and Ophthalmology 7 (2018).

20. Parrish RK., et al. "Visual function and quality of life among patients with glaucoma". Archives of Ophthalmology 115 (1997): 1447-1455.

21. Cesareo M., et al. "Visual disability and quality of life in glaucoma patients". Progress in Brain Research 221 (2015): 359-374.

22. Hu CX., et al. "What Do Patients With Glaucoma See? Visual Symptoms Reported by Patients With Glaucoma". The American Journal of the Medical Sciences 348 (2014): 403-409.

23. Liu JL., et al. "Smart System Contrast Sensitivity Measurements Compared With Standard Visual Function Measurements in Primary Open-Angle Glaucoma Patients". Journal of Glaucoma 26 (2017): 528-533.

24. Patodia Y., et al. "Clinical effectiveness of currently available low-vision devices in glaucoma patients with moderate-to-severe vision loss". Clinical Ophthalmology 11 (2017): 683-687.

Volume 4 Issue 6 June 2021

(C) All rights are reserved by Priyanka Sudhakar and Meena G Menon.

Citation: Priyanka Sudhakar and Meena G Menon. "Analysis of Visual Function and the Effectiveness of Assistive Devices in a Glaucoma Cohort at a Tertiary Eye Hospital". Acta Scientific Ophthalmology 4.6 (2021): 76-82. 